\title{
Clinical Implications of Adrenal Cancer
}

\author{
Paris A. Kosmidis
}

\subsection{Metastatic Tumors}

The adrenal gland is a significant target organ for metastasis in patients known to have cancer, i.e., the lung. CT is mostly done for initial diagnosis and staging of cancer in different organs and has contributed to the increased incidental finding of an adrenal mass $[1,2]$. This is an important finding for the decision-making process. It upgrades the stage of the disease and may change the decision for operation of the primary site. Lately, single metastatic lesion in the adrenal gland from cancers such as lung cancers may be removed with reasonable survival benefit for the patients.

PET-CT scan has facilitated the most accurate diagnosis of malignancy, and it is a desirable imaging test to be done prior to surgery. Certainly, in equivocal cases a biopsy is mandatory.

CT and/or MRI are very helpful imagine tests for response evaluation following treatment either surgery or chemotherapy and targeted treatment. Reappearance of a mass or progressively increasing mass declares progressive disease.

On the contrary, elimination of the size of the tumor means responsive disease. Unchanged mass declares stable disease. Careful evaluation of the tumor mass density is necessary to identify areas of necrotic tissue within the mass following treatment which is usually compatible with good response.

Ultrasound, CT, and MRI are very accurate and commonly used imagine tests for the followup of these patients $[1,2]$.

\subsection{Primary Tumors}

CT is the commonest used imagine test for diagnosis, staging, and follow-up of patients with primary adrenal gland tumors. MIBG scanning is a complementary test for staging pheochromocytoma $[1,2]$.

\section{References}

1. Blake MA, Cronin CG, Boland GW (2010) Adrenal Imaging. AJR 194(6):1450-1460

2. Taffel M, Haji-Manenian S, Nikolaidis P et al (2012) Adrenal imaging: a comprehensive review. Radiol Clin N Am 50(2):219-243

P. A. Kosmidis

Medical Oncologist,

Head, Medical Oncology Department,

Hygeia Hospital, Athens, Greece

e-mail: parkosmi@otenet.gr 